01

\title{
О двузначных волновых функциях в описании внутримолекулярных координатных движений
}

\author{
(C) А.В. Буренин \\ Институт прикладной фризики РАН, \\ 603950 Нижний Новгород, Россия \\ e-mail: buran@appl.sci-nnov.ru
}

Поступила в редакцию 14.05.2018 г.

Показано, что появление двузначных волновых функций при построении методами CNPI-группы классификации дискретного спектра координатных движений в молекулах с линейным остовом и двумя тождественными волчками является следствием физически некорректного определения действия операций симметрии на переменные конфигурационного пространства молекулы.

DOI: $10.21883 /$ OS.2018.09.46544.135-18

\section{Введение}

Хорошо известно [1], что при построении классификации дискретного спектра в нежестких молекулах с линейным остовом и двумя тождественными волчками с помощью полной ядерной перестановочно-инверсионной (CNPI) группы в описании внутренней динамики возникают двузначные вращательные, торсионные и колебательные волновые функции. Однако такое описание противоречит фундаментальному закону квантовой механики, согласно которому все координатные движения должны описываться однозначными волновыми функциями, а двузначные функции возникают только в описании спиновых движений квантовых систем с полуцелым спином [2]. В настоящей работе показывается, что противоречие с фундаментальным законом появляется из-за физически некорректного определения действия операций группы молекулярной симметрии (MS-группы, включающей из CNPI-группы лишь так называемые возможные элементы ${ }^{1}$ ) на переменные конфигурационного пространства молекулы. Соответственно некорректно задается действие этих операций на базисные функции гильбертова пространства дискретного спектра молекулы. Как будет показано в настоящей работе, это происходит потому, что CNPI-группа и, как следствие, MS-группа не содержат никакой информации о геометрической симметрии внутримолекулярной динамики, в то время как такая симметрия играет принципиальную роль [3]. В результате задание действия операций MS-группы плохо формализуется и является неоднозначным, причем выделить его физически корректный вариант очень сложно. Рассмотрение в работе проводится на примерах молекул с линейным остовом и двумя симметричными, плоскими и линейными тождественными волчками.

\footnotetext{
1 Данные элементы отвечают преобразованиям молекулы, которые могут быть реализованы с помощью рассматриваемых внутренних движений ядер [1].
}

\section{Молекулы с симметричными волчками}

Классический пример - молекула этана $\mathrm{C}_{2} \mathrm{H}_{6}$ в основном электронном состоянии. Показанная на рис. 1 ее равновесная конфигурация является шахматной и отвечает точечной группе $D_{3 d}$ [4]. Нужно отделить торсионное движение метильных волчков $\mathrm{CH}_{3}$ от поворота молекулы как целого вокруг линейного остова. Впервые на появление двузначных волновых функций в такой задаче при работе с MS-группой $G_{36}$ молекулы этана было указано в [5]. В [1] этому дается следующее объяснение. Конфигурационное торсионновращательное пространство имеет 4 координаты: $\theta, \varphi-$ полярные углы ориентации линейного остова; $\chi_{1}, \chi_{2}$ углы поворота двух метильных волчков вокруг этого остова. Для разделения вращательного и торсионного движений вводятся вращательный и торсионный углы, определенных по модулю $2 \pi$ :

$$
\chi=\left(\chi_{1}+\chi_{2}\right) / 2, \quad \gamma=\left(\chi_{1}-\chi_{2}\right) / 2 .
$$

При этом двугранный торсионный угол $\tau=\chi_{1}-\chi_{2}=2 \gamma$ определяется по модулю $4 \pi$. При повороте одного

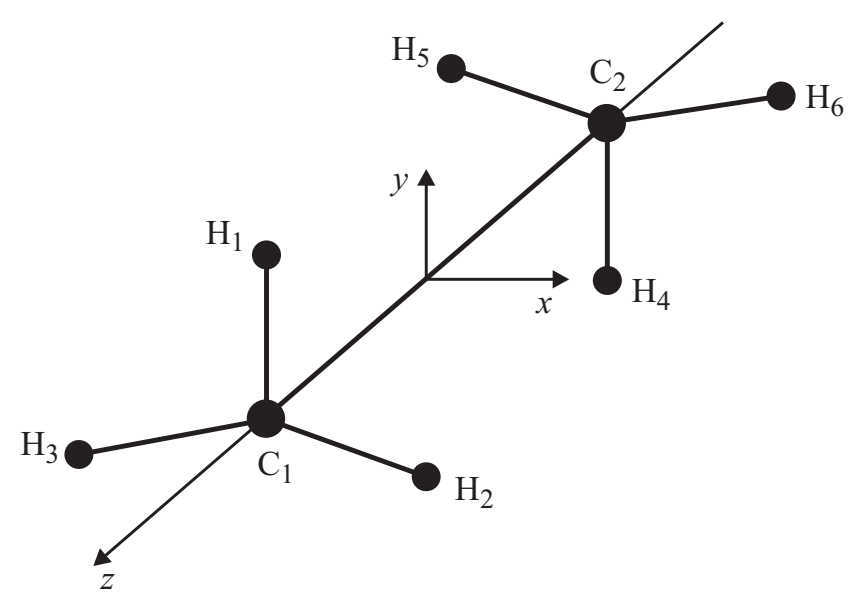

Рис. 1. Равновесная конфигурация молекулы этана (ядра $\mathrm{H}_{1}$ и $\mathrm{H}_{4}$ лежат в плоскости $\left.y z\right)$. 
волчка на угол $2 \pi / 3$ (преобразование (123) группы $G_{36}$ ) имеем для угла $\chi$

$$
(123) \chi=(123)\left(\chi_{1}+\chi_{2}\right) / 2=\left(\chi_{1}+\frac{2 \pi}{3}+\chi_{2}\right) / 2=\chi+\pi / 3 .
$$

Заменяя $\chi_{1}$ на $\chi_{1}+2 \pi$, что оставляет положение ядер в пространстве неизменным, вместо (2) получаем

$$
(123) \chi=\chi+4 \pi / 3 \text {. }
$$

Тот же результат имеет место и для угла $\gamma$. Такие же неопределенности возникают для всех элементов группы $G_{36}$. Поэтому в ней нельзя однозначно классифицировать вращательные, торсионные и даже колебательные функции. Чтобы обойти эту трудность, предлагается считать, что описываемые углами $(\chi, \gamma)$ и $(\chi+\pi, \gamma+\pi)$ конфигурации не одинаковы. В результате возникают двузначные волновые функции в описании этих движений, а группа $G_{36}$ заменяется ее двойной группой $G_{36}(E M)$. Понимая, что такое описание противоречит фундаментальному закону квантовой механики об однозначности волновых функций любых координатных движений, двузначные функции в [1] называются искусственными (artificial wave functions).

Фактически при работе с MS-группой задается действие элементов симметрии строгой задачи (перестановки тождественных ядер и их произведения с операцией пространственной инверсии) на координаты конфигурационных пространств ряда приближенных моделей, на основе которых вводятся сами понятия интересующих нас внутренних движений (в данном случае вращательного и торсионного). Оказывается, при этом очень важен правильный учет геометрической симметрии задачи. Однако такая информация в MS-группе отсутствует, что и приводит к физически некорректному описанию. Покажем это, используя правильное разделение торсионного и вращательного движений в молекуле этана, полученное в [3] методами цепочки групп, органично учитывающих геометрическую симметрию внутренней динамики молекулы.

Геометрическая симметрия гамильтониана внутреннего движения нежесткой молекулы этана $\mathrm{C}_{2} \mathrm{H}_{6}$ в основном электронном состоянии описывается расширенной точечной группой

$$
G_{72}=\left(c_{3 v} \times c_{3 v}\right) \wedge C_{I},
$$

где две группы $c_{3 v}$ определяются симметрией двух волчков $\mathrm{CH}_{3}$, а действующая на конфигурацию в целом операция инверсии $I$ учитывает тождественность волчков. Символ $\wedge$ означает полупрямое произведение $[3,6]$, возникающее вследствие некоммутативности элементов группы $c_{3 v} \times c_{3 v}$ и операции $I$. А именно

$$
(p \times q) I=I(q \times p),
$$

где $p \times q-$ произвольный элемент группы $c_{3 v} \times c_{3 v}$. Из (5) сразу следует, что $c_{3 v} \times c_{3 v}-$ это инвариантная подгруппа группы $G_{72}$, что является необходимым и достаточным условием записи последней в виде (4). Отметим, что группа $G_{72}$ включает точечную группу $D_{3 d}=C_{3 v} \times C_{I}$ как подгруппу. При этом реальная структура уровней дискретного спектра характеризуется полной вращательной подгруппой $H_{36}$ группы $G_{72} \times C_{i}$, где $C_{i}=(E, i)$ - группа пространственной инверсии. Изоморфная группе $G_{36}$ группа $H_{36}$ записывается в виде

$$
H_{36}=B_{6} \times D_{3} .
$$

Здесь $D_{3}-$ вращательная подгруппа группы $D_{3 d}$, а $B_{6}=B_{3} \wedge H_{2}$, где $B_{3}=\left(E \times E, c_{3} \times c_{3}^{2}, c_{3}^{2} \times c_{3}\right)$ и $\mathrm{H}_{2}=(E, I i)$. Запись (6) группы $H_{36}$ позволяет однозначно разложить торсионно-вращательное движение на торсионное и вращательное, определяемые элементами групп $B_{3}$ и $D_{3}$ соответственно. Важно, что элементы этих групп коммутируют между собой. Поэтому элементы группы $B_{3}$ не действуют во вращательном пространстве, а элементы группы $D_{3}-$ в торсионном ${ }^{2}$. Изящное решение, целиком определяемое геометрией задачи. Подчеркнем, что хотя элемент $C_{3}^{k}(k=1,2)$ группы $D_{3}$ может быть записан как $c_{3}^{k} \times c_{3}^{k}$, он не переводит молекулу из одного минимума эффективного ядерного потенциала в другой (первая операция $c_{3}^{k}$ действительно переводит молекулу в другой минимум, однако вторая операция возвращает ее в исходный минимум). Поэтому операция $c_{3}^{k} \times c_{3}^{k}$ не связывает независимые локальные минимумы и, как следствие, описывает обычное вращение молекулы как целого без барьера. Подчеркнем, что согласно данному рецепту разделения поворот одного волчка не является чисто торсионным движением. В частности, поворот $c_{3}^{1} \times E$ является суперпозицией торсионного движения $c_{3}^{2} \times c_{3}^{1}$ и вращения $C_{3}^{2}$ молекулы как целого.

Естественно, что рецепт разделения торсионного и вращательного движений на основе геометрической группы $H_{36}$ качественно отличается от рецепта разделения на основе MS-группы. Суть различия хорошо видна из сравнения действия элемента $(123)(456)$ группы $G_{36}$ и соответствующего ему элемента $C_{3}^{1}$ группы $H_{36}$ :

$$
\begin{aligned}
(123)(456) \chi & =\chi, \quad(123)(456) \tau=\tau+4 \pi / 3, \\
C_{3}^{1} \chi & =\chi+2 \pi / 3, \quad C_{3}^{1} \tau=\tau .
\end{aligned}
$$

Tо есть вращение молекулы как целого в MS-группе представляется в виде суперпозиции торсионных движений двух волчков. Естественно, что такое представление ошибочно, поскольку в отличие от торсионного движения вращение молекулы как целого в изотропном пространстве должно быть свободным.

\footnotetext{
2 При отсутствии коммутации ситуация меняется. Например, согласно (5), элемент $I i$ не коммутирует с элементами группы $B_{3}$. В результате элемент $I i$ нетривиальным образом, определяемым свойствами полупрямого произведения, действует в торсионном пространстве и полная симметрия торсионного движения описывается группой $B_{6}$ [3].
} 


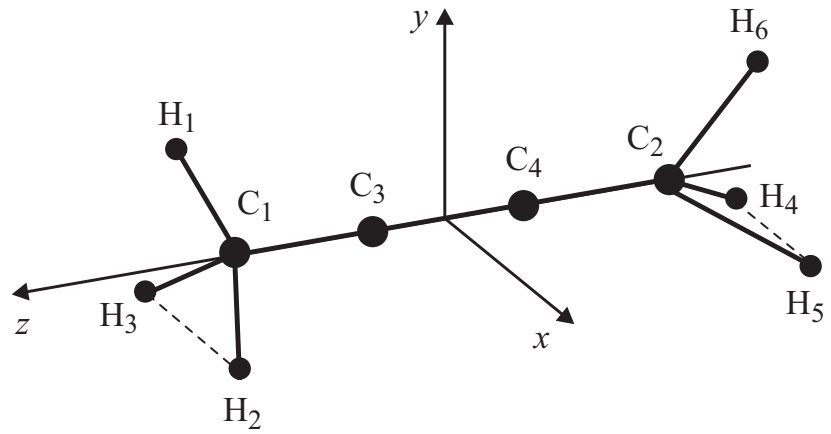

Рис. 2. Конфигурация молекулы диметилацетилена, отвечающая точечной группе $D_{3 h}$ (ядра $\mathrm{H}_{1}$ и $\mathrm{H}_{6}$ лежат в плоскости $\left.y z\right)$.

Другим полезным примером является молекула диметилацетилена $\mathrm{CH}_{3} \mathrm{C}_{2} \mathrm{CH}_{3}$ в основном электронном состоянии. Она дополнительно интересна тем, что торсионный барьер согласно исследованиям методами спектроскопии высокого разрешения $[7,8]$ равен всего $6.0 \mathrm{~cm}^{-1}$, и в качестве равновесной конфигурации возможна или шахматная или затененная. Показанная на рис. 2 затененная конфигурация отвечает точечной группе $D_{3 h}=C_{3 v} \times C_{S}$. Для перехода к шахматной конфигурации необходимо один из волчков повернуть вокруг оси $z$ на угол $\pi$. Заметим, что для равновесной шахматной конфигурации вершина торсионного барьера соответствует затененной конфигурации, и наоборот. Естественно возникает вопрос о следствиях в свойствах симметрии внутренней динамики нежесткой молекулы диметилацетилена при изменении типа ее равновесной конфигурации. При применении MS-группы ответ на него очень сложен, так как в ней нет информации о геометрии системы, и в обоих вариантах она одна и та же [1]. Другая ситуация при рассмотрении методами цепочки групп $[9,10]$. Геометрическая симметрия гамильтониана нежесткой молекулы диметилацетилена с затененной равновесной конфигурацией описывается расширенной точечной группой

$$
K_{72}=\left(c_{3 v} \times c_{3 v}\right) \wedge C_{S}
$$

В данном случае тождественность волчков учитывает действующая на конфигурацию в целом операция отражения $\sigma^{(x y)}$ группы $C_{S}$. Поэтому группа $K_{72}$ включает как подгруппу уже точечную группу $D_{3 h}$. Реальная структура уровней описывается полной вращательной подгруппой $M_{36}$ группы $K_{72} \times C_{i}$ :

$$
M_{36}=A_{6} \times D_{3} .
$$

Здесь $A_{6}=B_{3} \wedge C_{2}$, где $C_{2}=\left(E, C_{2}^{(z)}=\sigma^{(x y)} i\right)$. Хотя группы $M_{36}$ и $H_{36}$ изоморфны, их элементы имеют разную геометрическую интерпретацию. Наиболее важно, что теперь вращение молекулы как целого определяется элементами группы $D_{6}=C_{2} \times D_{3}$, причем элемент $C_{2}^{(z)}$ входит в группу $A_{6}$ и из-за геометрии задачи не коммутирует с элементами группы $B_{3}$, определяющими торсионное движение. Поэтому элемент $C_{2}^{(z)}$ нетривиальным образом, определяемым свойствами полупрямого произведения, действует и в торсионном пространстве [9]. Такое различие в группах $M_{36}$ и $H_{36}$ ведет к целому ряду важных изменений в классификации стационарных состояний и выражениях для эффективных операторов физических величин (включая эффективный гамильтониан) при рассмотренной смене типа равновесной конфигурации $[9,10]^{3}$.

\section{Молекулы с плоскими волчками}

В качестве примера наиболее часто рассматривается молекула этилена $\mathrm{C}_{2} \mathrm{H}_{4}$ в основном электронном состоянии. Соответствующая равновесная конфигурация плоская и принадлежит точечной группе $D_{2 h}$ [4]. Будем считать, что в связанной с молекулой подвижной системе координат эта конфигурация лежит в плоскости $y z$, а внутреннее вращение волчков ${ }^{4} \mathrm{CH}_{2}$ происходит вокруг оси $z$, проходящей через равновесные положения ядер С. Нежесткая молекула этилена описывается MS-группой $G_{16}$, которая в силу причин, аналогичных молекулам этана и диметилацетилена, заменяется ее двойной группой $G_{16}(E M)[1]$.

В методах цепочки групп реальная структура уровней молекулы этилена характеризуется изоморфной MS-группе $G_{16}$ группой $H_{16}$ [12]:

$$
H_{16}=U_{2} \times\left[\left(c_{2} \times c_{2}\right) \wedge C_{2}\right] .
$$

Внутренние вращения волчков задают элементы $c_{2}^{1} \times E$ и $E \times c_{2}^{1}$ группы $c_{2} \times c_{2}$, а вращения молекулы как целого элемент $C_{2}^{(z)}=c_{2}^{1} \times c_{2}^{1}$ группы $c_{2} \times c_{2}$, а также группы $U_{2}=\left(E, U_{2}^{(x)}=\sigma^{(y z)} i\right)$ и $C_{2}=\left(E, C_{2}^{(x)}=\sigma^{(y z)} I\right)$. Элемент $C_{2}^{(z)}$ аналогично элементу $C_{3}^{k}$ молекулы этана не связывает независимые локальные минимумы и поэтому описывает обычное вращение молекулы как целого без барьера. То есть группу $H_{16}$ невозможно представить как произведение групп, независимо описывающих торсионное и вращательное движения. Необходимо еще учесть, что из-за геометрии задачи вращение $C_{2}^{(x)}$ не коммутирует с $c_{2}^{1} \times E$ и $E \times c_{2}^{1}$. Поэтому действует и в торсионном пространстве, где такое действие легко находится из свойств полупрямого произведения. В торсионно-вращательных величинах (в волновых функциях и эффективных операторах физических величин) поведение торсионных частей по отношению к $c_{2}^{1} \times E$ и

\footnotetext{
${ }^{3}$ Из этих работ также следует, что применение MS-группы в силу указанных ее недостатков привело в [11] (см. также [1]) к неправильному ответу на вопрос о таких изменениях.

4 Заметим, что барьер внутреннего вращения для молекулы этилена в основном электронном состоянии довольно высок. Но традиционно рассмотрение такого движения прежде всего интересно с методологической точки зрения [1].
} 


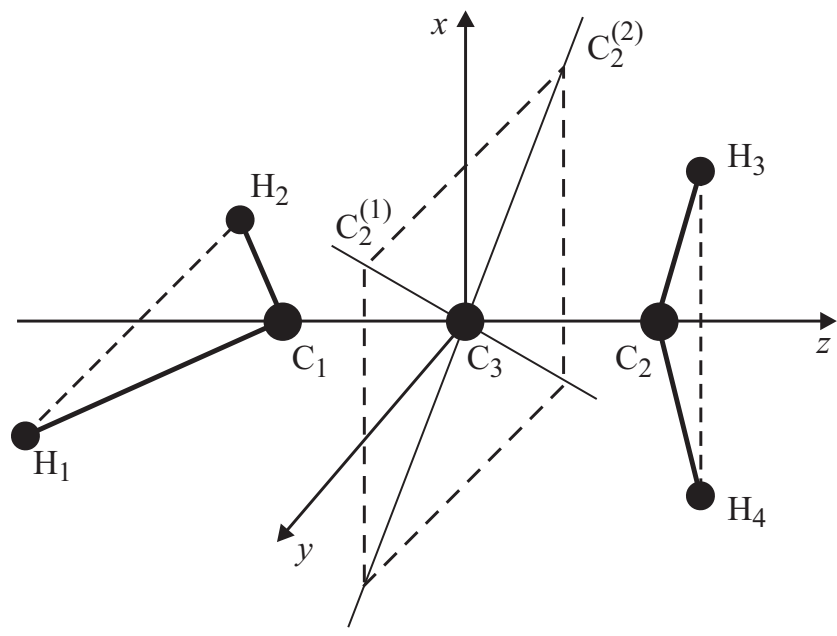

Рис. 3. Равновесная конфигурация молекулы аллена (волчки $\mathrm{C}_{1} \mathrm{H}_{1} \mathrm{H}_{2}$ и $\mathrm{C}_{2} \mathrm{H}_{3} \mathrm{H}_{4}$ лежат соответственно в плоскостях $y z$ и $x z$ ).

$E \times c_{2}^{1}$ однозначно задается неприводимым представлением группы $c_{2} \times c_{2}$. Тем самым определяется поведение вращательных частей относительно $C_{2}^{(z)}$. То есть геометрия задачи ведет к жесткой корреляции между торсионной и вращательной частями таких величин. В итоге получаем ответ на основной вопрос, в каких пространствах и каким образом действуют различные преобразования группы $H_{16}$. Данный ответ весьма нетривиален. Поэтому работая с MS-группой без информации о геометрической симметрии, вряд ли можно угадать его правильный аналог.

Полезно посмотреть на изменения в анализе при переходе к относящейся к тому же типу молекуле аллена $\mathrm{C}_{3} \mathrm{H}_{4}$. Дело в том, что молекула этилена является асимметричным волчком, и точечная группа $D_{2 h}$ содержит только невырожденные неприводимые представления. Молекула аллена, чья равновесная конфигурация в основном электронном состоянии приведена на рис. 3 , уже симметричный волчок и ее точечная группа $D_{2 d}[4]$ имеет двумерное неприводимое представление. Поэтому кроме торсионного вырождения уровней энергии, имеются вырождения, связанные с другими типами движения, и в том числе вращательные. Кроме того, равновесная конфигурация не плоская и в энергетическом спектре имеются инверсионные дублеты. Причем внутреннее вращение волчков $\mathrm{CH}_{2}$ их расщепляет, что довольно неожиданно для такого типа движения. Здесь этот эффект целиком объясняется плоскостностью волчков [13].

Несмотря на такое серьезное изменение геометрической симметрии равновесной конфигурации молекулы аллена по сравнению с молекулой этилена, MS-группа нежесткой молекулы аллена совпадает с таковой для этилена. Совсем иная ситуация в методах цепочки групп. Группа, описывающая реальную структуру уровней молекулы аллена, также представляется в виде (11), но теперь $[13]$

$$
U_{2}=\left[E \times E,\left(\sigma^{(y z)} \times \sigma^{(x z)}\right) i\right], \quad C_{2}=\left(E, C_{2}^{(1)}\right) .
$$

То есть по сравнению с молекулой этилена меняется геометрическая интерпретация элементов. В частности, $U_{2}$ уже не является группой преобразований молекулы как целого. Но при этом в качестве подгруппы, элементы которой задают вращение молекулы как целого, группа $H_{16}$ в случае молекулы этилена включает группу асимметричного волчка $D_{2}$, а в случае молекулы аллена - группу симметричного волчка $D_{4}$. Интересно, что различия в геометрии равновесных конфигураций молекул этилена и аллена таковы, что характеризующие их точечные группы даже не изоморфны. Однако расширенные точечные группы, включающие точечные группы в качестве подгрупп, и группы, описывающие реальную структуру уровней энергии, являются уже изоморфными, что подчеркивает однотипность этих молекул с точки зрения нежестких движений. Но при этом налицо качественные различия в их спектрах. Действительно, в молекуле этилена инверсионные дублеты отсутствуют, и поэтому в торсионных расщеплениях оба подуровня имеют одинаковое поведение относительно операции пространственной инверсии $i$. Причем расщепляется только половина вращательных уровней энергии, а остальные просто становятся двукратно вырожденными [12]. В то же время в молекуле аллена инверсионные дублеты имеются и именно они расщепляются вследствие торсионного движения, так как такое движение из-за плоскостности волчков приводит к реализуемости операции пространственной инверсии. В результате в расщеплениях подуровни имеют разное поведение относительно $i$, причем расщепляются все вращательные уровни [13]. Важно, что эти различия целиком объясняются разной геометрической интерпретацией элементов их расширенных точечных групп. Естественно, что правильно описать их на основе MS-группы, которая одна и та же для обеих молекул, не представляется реальным ${ }^{5}$.

\section{Молекулы с линейными волчками}

Классическим примером является молекула перекиси водорода НООН. Показанная на рис. 4 ее равновесная конфигурация в основном электронном состоянии принадлежит точечной группе $C_{2}=\left(E, C_{2}^{(x)}\right)$. Как следствие, молекула обладает стереоизомерами 6 . Их смешивание в жестких молекулах отсутствует, и вводят понятие „правой“ и ,левой“ модификаций молекулы с совпадающими энергетическими уровнями. В молекуле $\mathrm{HOOH}$

\footnotetext{
${ }^{5}$ Как уже ранее упоминалось, попытка на основе MS-группы описать изменения, связанные с разным выбором равновесных конфигураций молекулы диметилацетилена, привела к неправильному ответу. Причем несмотря на то, что задача значительно проще, поскольку точечные группы возможных равновесных конфигураций этой молекулы изоморфны.

6 Для наличия стереоизомерии требуется, чтобы точечная группа молекулы не имела несобственных преобразований [2].
} 


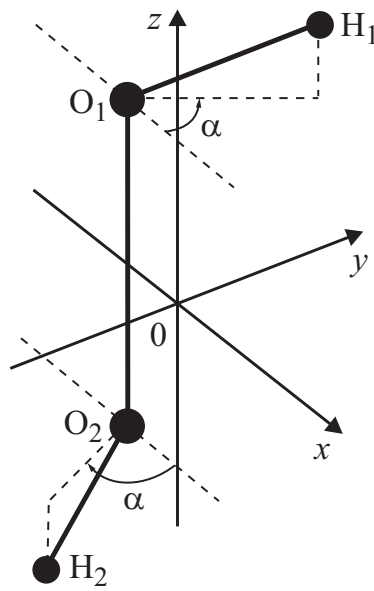

Рис. 4. Равновесная конфигурация молекулы перекиси водорода $(0-$ точка центра масс, $2 \alpha-$ двугранный угол между плоскостями $\mathrm{H}_{1} \mathrm{O}_{1} \mathrm{O}_{2}$ и $\left.\mathrm{H}_{2} \mathrm{O}_{2} \mathrm{O}_{1}\right)$.

структуры ОН туннелируют между энергетически эквивалентными положениями. Так как центр масс молекулы не лежит на химической связи ОО, нежесткое движение можно представлять как синхронное вращение структур вокруг оси $\mathrm{z}$ навстречу друг другу (цис-переход) или друг от друга (транс-переход). Естественно, что транс-переход является доминирующим [14]. Расширенная точечная группа, учитывающая геометрию таких нежестких движений, имеет вид [3]

$$
F_{8}=C_{2} \times C_{S} \times C_{S}^{\prime},
$$

где группа $C_{S}=\left(E, \sigma^{(y z)}\right)$ задает транс-переход, а группа $C_{S}^{\prime}=\left(E, \sigma^{(x z)}\right)-u u c$-переход ${ }^{7}$. Важно, что при отражении в плоскостях $y z$ и $x z$ меняется пространственное положение эффективного потенциала взаимодействия ядер (и, как следствие, положение равновесной конфигурации). То есть тождественные ядра не обмениваются местами друг с другом, а занимают новые положения в пространстве. Такие нежесткие движения в методах цепочки групп называются движениями необменного типа. Соответствующие им преобразования не являются элементами симметрии гамильтониана внутреннего движения молекулы. В результате $G_{8}$ это неинвариантная группа, в которой подгруппа симметрии гамильтониана совпадает с точечной группой $C_{2}$.

Реальная структура уровней энергии описывается полной группой $F_{8} \times C_{i}$, так как все ее элементы выражаются через операции, характеризующие рассматриваемые типы движений. В частности,

$$
i=\sigma^{(y z)} C_{2}^{(x)}=\sigma^{(x y)} C_{2}^{(y)} .
$$

Отсюда следует, что как транс-переход, так и цис-переход смешивают стереоизомеры. Всего группа $F_{8} \times C_{i}$ содержит 16 элементов.

\footnotetext{
${ }^{7}$ Вершине барьера для транс-перехода отвечает двугранный угол, равный $\pi$ (молекула лежит в плоскости $y z$ ), а для цис-перехода угол, равный 0 (молекула лежит в плоскости $x z$ ).
}

B качестве MS-группы при описании нежесткой молекулы перекиси водорода используется группа $G_{4}[1]$ :

$$
G_{4}=(E,(12)(34)) \times\left(E, E^{*}\right),
$$

где (34) - операция перестановки двух тождественных ядер $\mathrm{O}$, а $E^{*}$ - операция пространственной инверсии. Группа $G_{4}$ значительно беднее группы $F_{8} \times C_{i}$. В этом нет ничего удивительного, так как по самому своему определению MS-группа является группой симметрии гамильтониана внутримолекулярного движения. Поэтому она изоморфна подгруппе симметрии гамильтониана $C_{2} \times C_{i}$ группы $F_{8} \times C_{i}$. Из-за бедности MSгруппы транс-переход приходится задавать элементом $(12)(34) E^{*}$, хотя $E^{*}$ является связанной со свойствами пространства внешней операцией симметрии и поэтому не может иметь отношения к заданию нежестких движений молекулы ${ }^{8}$. Но просто нет другого варианта. Еще более странно задание ицс-перехода с помощью элемента $E^{\prime}(12)(34) E^{*}$, где $E^{\prime}-$ образующий элемент перехода от группы $G_{4}$ к ее двойной группе $G_{4}(E M)$, для которого выполняется условие $E^{\prime 2}=E$. То есть для задания нежесткого движения используется совершенно абстрактный элемент $E^{\prime}$, и в этом плане ситуация серьезно отличается от молекул с симметричными и плоскими волчками. Там нежесткие движения задаются перестановками тождественных ядер. Это можно сделать по той простой причине, что геометрические преобразования, задающие нежесткие движения, не меняют пространственное положение эффективного ядерного потенциала, а движение ядер в этом потенциале сводится к обмену тождественных ядер местами друг с другом. Такие преобразования являются элементами симметрии гамильтониана, а отвечающие им движения в методах цепочки групп называются движениями обменного типа. При анализе подобных нежестких движений в молекулах с линейным остовом и двумя тождественными волчками двойная MS-группа появляется только потому, что некорректно определяется действие операций симметрии MS-группы, заданных перестановками тождественных ядер, на переменные конфигурационного пространства молекулы. Последнее связано с отсутствием информации о геометрии внутренних движений. Однако в молекуле перекиси водорода нежесткие движения относятся к необменному типу, которым нельзя сопоставить перестановку тождественных ядер. Здесь ярко проявляется принципиальный недостаток подхода на основе MS-группы. Приходится привлекать для задания транс-перехода элемент $E^{*}$, а для задания цисперехода элементы $E^{*}$ и $E^{\prime}$. То есть двойная группа используется уже на этапе задания нежестких движений. Такая схема анализа свойств симметрии вообще лишена какого-либо физического смысла.

\footnotetext{
${ }^{8}$ Другое дело, что полная координатная волновая функция стационарного состояния изолированной молекулы должна иметь определенное поведение относительно этой операции.
} 


\section{Заключение}

Проведенный в этой работе анализ молекул с линейным остовом и двумя тождественными волчками наглядно демонстрирует значение геометрической симметрии для правильного описания их внутренней динамики. Важно, что в настоящее время такую симметрию в случае нежестких молекул позволяют адекватно учитывать только методы цепочки групп.

\section{Список литературы}

[1] Bunker P.R., Jensen P. Molecular symmetry and spectroscopy, NRC Research Press, Ottawa, 1998. 750 p. Перевод: Банкер Ф., Йенсен П. Симметрия молекул и спектроскопия. М.: Мир, 2004. 764 с.

[2] Ландау Л.Д., Лифииц Е.М. Квантовая механика. М.: Наука, 1989. 767 с.

[3] Буренин А.В. Симметрия квантовой внутримолекулярной динамики. 3-е изд., Н.Новгород: ИПФ РАН, 2012. 416 c. Burenin A.V. Symmetry of intramolecular quantum Dynamics. Berlin/Boston: Walter de Gruyter, 2012. 430 p.

[4] Свердлов Л.М., Ковнер М.А., Крайнов Е.П. Колебательные спектры многоатомных молекул. М.: Наука, 1970. $560 \mathrm{c}$.

[5] Hougen J.T. //Can. J. Phys. 1964. V. 42. P. 1920.

[6] Эллиот Джс., Добер П. Симметрия в физике. Т. 2. М.: Мир, 1983. $412 \mathrm{c}$.

[7] Pliva J., Pine A.S., Civis S. // J. Mol. Spectrosc. 1996. V. 180. P. 15.

[8] Lauro C., Bunker P.R., Johns J.W.C., McKellar A.R.W. // J. Mol. Spectrosc. 1997. V. 184. P. 177.

[9] Буренин А.В. // Опт. и спектр. 2016. Т. 121. С. 683.

[10] Буренин А.B. // Опт. и спектр. 2017. Т. 123. С. 3.

[11] Bunker P.R., Lauro C. // Chem. Phys. 1995. V. 190. P. 159.

[12] Буренин А.В. // Опт. и спектр. 2017. Т. 123. С. 834.

[13] Буренин А.В. // Опт. и спектр. 2017. Т. 123. С. 841.

[14] Внутреннее вращение молекул / Под ред. В.Дж. ОрвиллТомас. М.: Мир, 1977. 511 с. 